Artículo

\title{
Las creencias de los profesores universitarios sobre evaluación del aprendizaje
}

\author{
Professors' beliefs about evaluation of learning \\ Nelson Rubén Martínez Reyes* \\ nelson.martinez@udb.edu.sv
}

ISSN 1996-1642, Editorial Universidad Don Bosco, año 7, No.12, Julio-Diciembre de 2013, pp.45-66

Recibido: 25 de agosto de 2013 Aceptado: 13 de septiembre 2013

\section{Resumen}

Este artículo presenta los hallazgos de la investigación realizada acerca de las creencias que sobre evaluación del aprendizaje tienen los profesores de la Universidad Don Bosco. Este es un estudio cualitativo que precisamente buscaba caracterizar las creencias de los profesores y ver cómo estas se vinculan a modelos educativos y evaluativos tradicionales o constructivistas. El estudio parte de la premisa que muchas de las creencias que los profesores tienen sobre enseñanzaaprendizaje-evaluación guían de alguna forma su práctica educativa.

Palabras clave: creencias, evaluación, profesor, aprendizaje, educación
Abstract

This paper shows the findings of the research regarding the teachers' beliefs about assessment at Universidad Don Bosco. This is a qualitative research whose goal was precisely to characterize the teachers' beliefs and how these beliefs relate to either traditional or constructivist educational and evaluative models. The research stands on the premise that a lot of the teachers' beliefs about teachinglearning-assessment eventually guide the teacher's instructional practice in the classroom.

Keywords: beliefs, assessment, teacher, learning, education

* Candidato a Doctor en Educación por la Universidad de Costa Rica. Académico e investigador, Escuela de Educación de la Universidad Don Bosco

Para citar este artículo: Martínez Reyes, N. R. (2013). Las creencias de los profesores universitarios sobre evaluación del aprendizaje. Diá-logos 12, 45-66. 


\section{Introducción}

Abordar la evaluación del aprendizaje es abordar un tema complejo, polisémico, impreciso, que es además controversial y contradictorio. Sin embargo, a pesar de todo eso, la evaluación no es un tema aislado, esporádico o infrecuente, sino más bien es un tema central a toda acción educativa y es una actividad que está muy presente en todo proceso formativo en la educación formal y sistemática. Es una actividad que está tan arraigada a los procesos de enseñanza y aprendizaje que es prácticamente imposible pensar en cualquier forma de educación sin su evaluación.

Como actividad humana que es, la evaluación del aprendizaje está adscrita al pensamiento y práctica del profesor, y en éste, la concepción de evaluación ha tenido significados y sentidos muy diferentes en distintos tiempos y ámbitos geográficos. Como parte de su desarrollo sociohistórico, la concepción de evaluación y su práctica han evolucionado en razón de los cambios de paradigmas, las teorías psicológicas y del aprendizaje, el concepto de educación, y en la misma medida que han evolucionado las instituciones educativas.

Como actividad adscrita al profesor, la evaluación queda condicionada en un primer momento por su forma particular de ver y entender la educación, el conocimiento, la enseñanza, el aprendizaje y la escuela. La práctica evaluativa pasa por el filtro de la visión, prejuicios, limitaciones y carencias propias del profesor así como a sus talentos, habilidades y conocimientos adquiridos tanto a través de la práctica y la experiencia como por su formación docente. Es decir, las actuaciones del profesor en los procesos de enseñanza y evaluación tienen como base, casi siempre implícitas, sus creencias y concepciones y el conocimiento profesional, de carácter teórico-científico, adquirido en su formación docente.

El hecho es que los profesores tienen un sistema de creencias a los cuales recurren para entender el mundo, actuar en él y resolver los problemas que este le plantea. Estos sistemas de creencias actúan no solo en la vida cotidiana, sino también son aplicados en el ámbito profesional y sirven para actuar sobre las situaciones de enseñanza, aprendizaje y evaluación. Los profesores a menudo recurren a sus creencias sobre todo cuando el conocimiento de algo es incierto, y toman preponderancia ante la falta de conocimientos específicos ya sea porque el profesor carece de formación o tiene una formación deficiente, en este caso, en el área de la evaluación. En este marco de las creencias se plantea el estudio de "Las creencias de los profesores universitarios sobre

Las creencias de los profesores universitarios sobre evaluación 46. del aprendizaje evaluación del aprendizaje", estudio desarrollado con los profesores de grado de Universidad Don Bosco en El Salvador. 


\section{El problema de investigación}

Identificar y caracterizar las creencias de los profesores sobre evaluación, su sentido y propósito -creencias que posteriormente se ponen en práctica en diversas actividades de evaluación y se traducen en calificación- permite develar no solo sus fortalezas, sino también sus debilidades y carencias de formación teórica-docente en el área de evaluación. Pero además permite explicar, al menos parcialmente, su pensamiento con respecto a una actividad que tiene gran incidencia en la calidad educativa-académica de la universidad, ya que la forma particular de realizar la evaluación, su rigurosidad y sistematización incide tanto en los altos índices de reprobación y deserción como en la relativa facilidad de aprobación de los cursos y carreras.

Además, ha de recordarse que la evaluación tiene incidencias y consecuencias directas en los estudiantes ya que esta cumple con una función certificadora que da cuenta del rendimiento académico del estudiante y es el referente que sirve de base para aprobar y reprobar. Tiene implicaciones que trascienden a una dimensión social ya que es a través de la evaluación que los profesores, las instituciones dan cuenta a la sociedad de la calidad formativa con que estos se integran a la sociedad. El punto de partida de todo este entramado de prácticas, incidencias y consecuencias lo constituyen las creencias y las concepciones sobre evaluación.

El punto de partida del presente estudio es que existe un desconocimiento del pensamiento de los profesores con respecto a la evaluación del aprendizaje. A este momento se desconoce qué creencias subyacen las prácticas de evaluación del aprendizaje de los profesores de la Universidad Don Bosco y con este estudio se busca contestar la pregunta ¿Cuáles son las creencias que los profesores tienen sobre evaluación del aprendizaje, cómo conciben la evaluación, qué propósito y sentido le dan? El objetivo del estudio era caracterizar las creencias de los profesores sobre evaluación del aprendizaje de tal manera que se pudiera reconstruir su concepción, propósito y sentido. Las preguntas que se sirvieron de guía para la presente investigación son:

1. ¿Qué entienden o cómo conceptualizan los profesores de la Universidad Don Bosco la evaluación del aprendizaje? ¿Cuál es la concepción de evaluación que tienen los profesores?

2. ¿ ¿Tiene la evaluación algún sentido para los profesores? De ser así ¿Cuál es el sentido de la evaluación del aprendizaje que ellos le dan?

3. Según los profesores, ¿Cuál es propósito o finalidad de la evaluación del aprendizaje?

4. ¿Con qué teoría del aprendizaje se identifican las más las creencias sobre evaluación de los profesores? 
5. ¿Cuál es la formación profesional sobre evaluación del aprendizaje del profesor?

\section{Marco teórico}

La evaluación del aprendizaje, como práctica y como disciplina, así como el tema de las creencias tienen ya una tradición de estudio dentro de las ciencias de la educación. Las creencias, un tema adscrito a la dimensión del pensamiento del profesor que también incluye las concepciones y conocimiento profesional, han venido siendo investigadas desde hace mucho tiempo lo cual constituye una muestra del interés científico y pedagógico que existe por conocer y reconocer que lo que el profesor cree y sabe, tienen incidencia directa en su práctica pedagógica. Es así que el tema de las creencias ya estaba en la mesa de estudio desde los treinta con Dewey (1933) y se retoma en los ochenta con Shulman (1996) con el pensamiento del profesor. Pajares (1992, 1993), Ponte (1999), Dilts (1996, 1997), Flores (1998), Gallego (1991), McKay y Fanning (1993), Vicente (1995), Bouvet (1999), Thompson (1992) son algunos de los autores, que durante la década de los noventa, estudiaron, construyeron y consolidaron el constructo de las creencias.

Las creencias (y a menudo también las concepciones) de los profesores han sido investigadas en relación con el proceso de enseñanza-aprendizaje y el currículo (Serrano, 2010; Ponte, 1999; Pajares, 1992; Gómez, 2008; Richardson, 1996; Contreras, 2009). También se han investigado con relación a ciertas disciplinas, especialmente matemáticas y ciencias (Contreras, 2009; GómezChacón, 2003; Faria, 2008; Moreno y Azcárate, 2003; Mora y Barrantes, 2008; García, Azcárate, y Moreno, 2006; Moreno y Azcárate, 2003. Con relación a la evaluación del aprendizaje, las creencias han sido poco investigadas (Prieto y Contreras, 2008; Prieto, 2008; Vergara, 2011; Mendoza, 1998; Rico, 1995; Alonso y otros, 1995; López, R. 2001).

\section{Las creencias}

La comprensión de las creencias pasa por un abanico de términos dado que en algunos momentos, algunos autores las han denominado con otros nombres. Las creencias han sido abordadas indistintamente como actitudes, valores, juicios, axiomas, opiniones, ideologías, percepciones, preconcepciones, disposiciones, procesos mentales internos, reglas de la práctica o principios prácticos (Prieto y Contreras, 2008).

Las creencias constituyen verdades personales derivadas de la experiencia o de la fantasía, con un fuerte componente afectivo y evaluativo, se manifiestan en las declaraciones verbales, escritas o en las acciones y, por lo tanto, condicionan los procesos de decisión (Pajares, 1992). Según Gómez-Chacón (2003), las 
creencias son parte del conocimiento subjetivo, pertenecen al dominio cognitivo y están compuestas por elementos afectivos, evaluativos y sociales,

son estructuras cognitivas que permiten al individuo organizar y filtrar las informaciones recibidas, y que van construyendo su noción de realidad y su visión del mundo. Las creencias constituyen un esquema conceptual que filtra las nuevas informaciones sobre la base de las procesadas anteriormente, cumpliendo la función de organizar la identidad social del individuo y permitiéndole realizar anticipaciones y juicios acerca de la realidad. (pp. 234-235).

De acuerdo con Carr y Kemmis (1998), las creencias no necesitan ser consensuadas para ser consideradas válidas, como tampoco requieren de reglas lógicas para determinar su correspondencia con situaciones reales. De tal manera que las creencias se constituyen en verdades idiosincráticas que no requieren una condición de verdad contrastada, dado que representan datos, supuestos y opiniones propias o transmitidas por otros y surgidas desde los saberes del sentido común.

Dentro del presente estudio se entiende que las creencias son:

conocimientos subjetivos, poco elaborados, generados a nivel particular por cada individuo para explicarse y justificar muchas de las decisiones y actuaciones personales y profesionales vividas. Las creencias no se fundamentan sobre la racionalidad, sino más bien sobre los sentimientos, las experiencias y la ausencia de conocimientos específicos del tema con el que se relacionan, lo que las hacen ser muy consistentes y duraderas para cada individuo (Moreno y Azcarate, 2003).

Pajares (1992) apunta las características de las creencias, de las cuales sólo se retoman algunas: (1) Las creencias se forman temprano y tienden a autoperpetuarse aún a pesar de las contradicciones causadas por la razón, el tiempo, la educación escolar, o la experiencia. (2) El conocimiento y las creencias están indisolublemente unidos, pero la fuerte naturaleza afectiva, evaluativa y episódica de las creencias las convierten en un filtro a través del cual se interpretan los nuevos fenómenos. (3) Por su propia naturaleza y origen, algunas creencias son más incontrovertibles que otras. (4) El cambio de una creencia durante la adultez es un fenómeno relativamente raro. (5) Los individuos tienden a sostener una creencia basada en conocimiento incorrecto e incompleto, aún después que se les han dado explicaciones científicas correctas. (6) Las creencias de los individuos afectan fuertemente su conducta. (7) Las creencias sobre la enseñanza están bien establecidas al momento que un estudiante va a la universidad. 
En el caso de los profesores, de acuerdo con Prieto (2008), las creencias han sido configuradas tanto por sus funciones y sentidos definidos externamente, como por lo que ellos mismos han percibido desde sus intercambios cotidianos escolares pasados y presentes, a la vez que concurren factores externos tales como el tiempo, los recursos disponibles, el tamaño de la clase y su formación profesional. Además, los profesores no construyen sus creencias de manera intencionada y consciente, sino que las han internalizado en sus estructuras cognitivas y emocionales de manera inconsciente, como resultado de una gran diversidad de experiencias articuladas (Prieto y Contreras, 2008).

Es necesario reconocer que, frecuentemente las creencias están tan fuertemente arraigadas en el inconsciente de los profesores que les hace difícil reconocer su existencia, pero acuden a ellas como respuestas válidas, especialmente cuando se ven enfrentados a situaciones desconocidas o confusas (Pajares, 1992). Igualmente, a menudo los profesores piensan que sus creencias son conocimiento profesional, lo cual dificulta su identificación y revisión crítica (Prieto, 2008). De la misma manera, es importante destacar que las creencias son los referentes a los que acuden los profesores de manera consciente o inconsciente para organizar y tomar las decisiones que guiarán las elecciones de las estrategias docentes y sus respuestas a las diferentes preocupaciones y prioridades (Durán, 2001; Ponte, 1999).

\section{La evaluación y el aprendizaje}

Aunque la base teórica de la evaluación es amplia, y no es propósito de este estudio desarrollarla, si resulta relevante enfatizar algunos aspectos que le dan su propia identidad y valor y que son pertinentes para el presente estudio. El punto de partida es definir qué se entiende por evaluación. Esto ya plantea un reto ya que dicho concepto es complejo y polisémico.

A la evaluación se le han asociado significados como estimar, valorar, atribuir el valor de algo, apreciar, justipreciar. En un sentido más cotidiano y práctico, pero sobre todo, más reduccionista, se ha entendido la evaluación como medición y calificación, y evaluar como sinónimo de examinar. Además, casi todo acercamiento a la evaluación ha estado marcado por una dimensión técnica-instrumental que deja por fuera una serie de aspectos consustanciales a la evaluación y por tanto sesga su conceptualización en función de dicha instrumentalización o de cierto paradigma o teoría educativa. A menudo dichas concepciones van desde los enfoques más tradicionales hasta los más constructivistas. Por eso aquí se propone que la evaluación del aprendizaje es

Las creencias de los profesores universitarios sobre evaluación 50. del aprendizaje
La actividad humana desarrollada dentro de los contextos educativos formales, generalmente ejercida por el profesor sobre el estudiante, a 
través de la cual se da significado a la acción educativa, se asignan calificaciones y se hacen valoraciones con respecto al aprendizaje del estudiante y para lo cual se usan diversos procedimientos e instrumentos que a menudo están orientados por un currículo, la institución educativa y el pensamiento del profesor.

Para González (2002), la evaluación del aprendizaje es el proceso y el resultado de juzgar la valía de un objeto o fenómeno de la realidad, el aprendizaje en este caso, en sus características esenciales, sus manifestaciones particulares de acuerdo con criterios de referencia pertinentes a la naturaleza del propio objeto. Siempre bajo la línea de análisis de González (2000), la evaluación tiene como propósito valorar tanto los procesos como los resultados de dicho aprendizaje de tal manera que sirvan para regular, valorar y orientar la actividad de enseñanzaaprendizaje y así lograr su finalidad formativa.

La evaluación se considera un proceso en tanto que su desarrollo implica una serie de acciones sucesivas que van desde el establecimiento de los objetivos de aprendizaje en la planificación hasta la presentación de los resultados a los estudiantes o padres de familia, pasando en el medio por muchas otras acciones eslabonadas. Además, se considera un proceso -o subprocesosubsumido dentro de otro proceso, en el sentido que la evaluación es parte del proceso de enseñanza-aprendizaje, un proceso más amplio y más complejo, al cual corre paralelamente y del cual es parte consustancial.

El objeto de la evaluación lo constituye el aprendizaje. Es decir, aquellos comportamientos, actitudes, afectos, saberes, competencias adquiridas y desarrolladas por el estudiante y que han sido previamente seleccionadas en el currículo, plasmados en la planificación didáctica y desarrollaos en la actividad educativa. El aprendizaje ha de entenderse como el propósito de formación que no está adscrito exclusivamente a la dimensión cognitiva, sino también a las afectivas, psicomotora, actitudinal y volitiva.

Con respecto al sentido de la evaluación del aprendizaje, éste no sólo consiste en proporcionar información respecto a los aprendizajes de los estudiantes, sino también en proporcionar indicios sobre la eficacia de la enseñanza (Jackson, 2002). El sentido no sólo está orientado a identificar necesidades y detectar dificultades, sino mas bien a recuperar y reorientar el pleno sentido formativo del proceso educativo (Prieto y Contreras, 2008). En general, se entiende que el sentido y la función de la evaluación es regular la actividad que en este caso es la actividad de enseñanza aprendizaje, con el fin de mejorarla. 


\section{El método}

Indagar sobre las creencias de los sujetos es indagar en las construcciones humanas, en los valores y significados particulares que los sujetos le dan a los fenómenos. De ahí que para poder acceder a ellos se ha de hacer a través de formas y estrategias que permitan los sujetos expresar esos significados bajo sus propios referentes, sin más limitantes que las que da el lenguaje. De ahí que el enfoque metodológico sea de corte cualitativo.

\section{Las estrategias e instrumentos de recolección de la información}

El grupo focal Por eso se optó en este estudio por el grupo focal como una técnica y estrategia a través de la cual los profesores, sin necesidad de buscar consenso o tratar de imponer criterio, pueden expresar y desarrollar sus ideas. Se desarrollaron cuatro grupos focales, formados con base a los criterios de facultad como categoría natural ya constituida en la institución y al de la cantidad de profesores en cada facultad para conformar los grupos focales de alrededor de ocho participantes. En el grupo focal se plantearon tres preguntas básicas: La primera relacionada al cómo ellos conciben la evaluación del aprendizaje, la segunda referida al sentido que le dan a la evaluación y la tercera al propósito de la misma.

El cuestionario Igualmente se utilizó el cuestionario como un instrumento para obtener los datos demográficos de los participantes y para hacer preguntas puntuales con respecto a las creencias de los profesores sobre evaluación. El instrumento incluía preguntas enmarcadas dentro de las categorías de (a) concepción, (b) sentido, y propósito de evaluación, que son las mismas categorías de análisis del gripo focal. Estos datos igualmente fueron triangulados con las opiniones expresadas en el grupo focal.

Procesamiento de los datos La información obtenida de los cuatro grupos focales fue grabada y transcrita. Posteriormente fue procesada a través de procedimientos analíticos recursivos que incluyeron (1) una reducción de los datos a través de códigos descriptivos e interpretativos; (2) exposición de los datos en cuadros y matrices; y (3) luego, a través de procesos de análisis inductivos, la extracción de conclusiones de acuerdo con las categorías de análisis, los patrones y la consistencia de los datos obtenidos.

Igualmente se usó el método de la triangulación se triangularon confrontaron los datos obtenidos de de los dos instrumentos como elementos para confirmar y consolidar los hallazgos. 
Población y muestra La población dentro del perfil de la población objeto de estudio, esta debía cumplir con los criterios de ser profesor tiempo completo y estar impartiendo, al momento del estudio, al menos un curso regular dentro del ciclo académico. Se excluyeron los profesores o instructores de talleres o cursos completamente prácticos, los de prácticas de campo y los profesores de la escuela de educación. Al momento de la investigación, la Universidad Don Bosco, en sus cinco facultades que la conforman, tenía ochenta y siete profesores tiempo completo que cumplían con dicho perfil.

La muestra Muestreo estratificado proporcional de los profesores que cumplían con el perfil, todos debían tener la misma posibilidad de ser seleccionados pero su representación en número debía ser proporcional al número de profesores por escuela y facultad. Por eso se seleccionó una muestra de acuerdo con el muestreo estratificado proporcional, aunque este no es un estudio cuantitativo, de donde se obtuvieron 27 profesores tiempo completo. La muestra y su representación proporcional queda ilustrada en la Tabla 1.

Tabla 1: Población y muestra para la formación de los cuatro grupos focales.

\begin{tabular}{|c|c|c|c|c|c|}
\hline Facultad & $\begin{array}{l}\text { No. Profesores } \\
\text { tiempo } \\
\text { completo }\end{array}$ & Escuelas & $\begin{array}{l}\text { No. Profesores } \\
\text { entrevistados } \\
\text { por escuela }\end{array}$ & Muestra & $\%$ \\
\hline \multirow{5}{*}{$\begin{array}{l}\text { Ciencias y } \\
\text { Humanidades }\end{array}$} & \multirow{5}{*}{25} & Teología & 2 & \multirow{5}{*}{8} & \multirow{5}{*}{32} \\
\hline & & Diseño gráfico & 2 & & \\
\hline & & Comunicaciones & 2 & & \\
\hline & & Idiomas & 2 & & \\
\hline & & Educación & 0 & & \\
\hline \multirow{5}{*}{ Ingeniería } & \multirow{5}{*}{28} & Electrónica & 2 & \multirow{5}{*}{8} & \multirow{5}{*}{25.5} \\
\hline & & Industrial & 2 & & \\
\hline & & Computación & 2 & & \\
\hline & & Biomédica & 1 & & \\
\hline & & Eléctrica & 1 & & \\
\hline \multirow[t]{2}{*}{ Economía } & \multirow[t]{2}{*}{9} & $\begin{array}{l}\text { Administración } \\
\text { de empresas }\end{array}$ & 2 & \multirow[t]{2}{*}{3} & \multirow[t]{2}{*}{33.3} \\
\hline & & Contaduría & 1 & & \\
\hline $\begin{array}{l}\text { Estudios } \\
\text { tecnológicos }\end{array}$ & 5 & $\begin{array}{l}\text { Técnico en } \\
\text { mantenimiento } \\
\text { Aeronáutico }\end{array}$ & 2 & 2 & 40 \\
\hline $\begin{array}{l}\text { Ciencias de la } \\
\text { rehabilitación }\end{array}$ & 4 & Órtesisi y prótesis & 3 & 3 & 75 \\
\hline $\begin{array}{l}\text { Dirección de } \\
\text { Ciencias Básicas }\end{array}$ & 16 & & 3 & 3 & \\
\hline Total & 87 & & 27 & 27 & 31 \\
\hline
\end{tabular}




\section{Los hallazgos}

El grupo focal permitió que los profesores expresaran sus ideas libremente. De esas ideas expresadas verbalmente, que fueron grabadas, transcritas, procesadas e interpretadas siguiendo el modelo antes descrito, surgieron los hallazgos que se presentan a continuación.

\section{Formación en evaluación y conocimiento profesional}

El primer hallazgo es que de los veintisiete profesores entrevistados, dieciocho (66.7\%) han cursado la asignatura Evaluación del aprendizaje como materia de estudio dentro de un plan de formación docente. Esto implica que un porcentaje bastante significativo de dicha muestra debería haber consolidado un conocimiento profesional o una base teórica bastante sólida con respecto a la naturaleza, sentido, propósito, función y otros aspectos importantes de esta disciplina de estudio. Dicho conocimiento profesional o formación teórica debería prevalecer sobre las creencias y concepciones de la evaluación a la hora de expresar sus argumentos y opiniones profesionales. Como se evidencia a continuación, el conocimiento profesional sobre evaluación del aprendizaje no prevalece sobre las creencias.

\section{La concepción de evaluación}

Un objetivo central del estudio era precisamente develar la concepción de evaluación, develar lo que entienden y creen qué es la evaluación. A ese respecto, el estudio encontró en un primer momento, que los profesores tienen ideas o nociones claras que sirven de indicadores, estas se explican a continuación:

Medición y cuantificación La primera es que los profesores tienen la concepción de evaluación como medición y cuantificación. Entienden que la evaluación está fuertemente asociada a la medición y la cuantificación. Esta es la creencia más consistente; la tendencia más presente, más evidente y más sólida con respecto a la conceptualización de la evaluación por parte del profesor. Ellos creen que la evaluación es "medir" o "una forma de medir". De la misma forma usan términos como "cuantificar", "cuánto", "que tanto", "en qué medida"... los alumnos han aprendido.

Yo creo que la evaluación es una forma de medir y lo que nosotros hacemos en un curso, es que planteamos objetivos y con esos objetivos lo que nosotros esperamos de los estudiantes, entonces en la evaluación estamos midiendo eso.

Básicamente es medir el conocimiento que ha adquirido un estudiante o cualquier persona en un proceso educativo, eso es. 
Parte del proceso enseñanza-aprendizaje que permite al alumno demostrar el nivel de aprendizaje que ha logrado, por parte del educador mide lo alcanzado de acuerdo a los contenidos.

Cuantificar en alguna medida los conocimientos adquiridos por los estudiantes.

Con la evaluación queremos ver qué tanto el estudiante ha logrado captar sobre la parte que corresponde a esa asignatura.

Medición y cuantificación tienen significados muy parecidos. Tanto la medición como la cuantificación implican una noción numérica y un proceso de cálculo. Tanto la medición como la cuantificación se expresan con números, con cantidades que a menudo son el resultado de operaciones matemáticas.

Verificación y comprobación Una segunda idea o noción expresada por los profesores es la de evaluación entendida como verificación y comprobación. Esta forma de entender la evaluación, aunque no tan consistente como la anterior, expresa la idea que la evaluación es una forma o un medio a través del cual los profesores comprueban y verifican los avances/conocimientos/ aprendizajes de los alumnos. Es decir se entiende la evaluación como una constatación a través de la cual el alumno demuestra sus avances y aprendizajes. Esta constatación implica que anteriormente se previó o planeó ciertos logros, avances o aprendizajes los cuales son verificados posteriormente a través de la evaluación. En sus palabras para conceptualizar la evaluación destacan:

Evaluación es una forma de verificar si el conocimiento que se quiso transmitir realmente se transmitió en el alumno, verificar si realmente ... se comprueba ese proceso y se comprueba verificando si tiene ese conocimiento ... la evaluación es verificar si ese proceso tuvo éxito o no en el alumno.

Yo considero que la evaluación del aprendizaje es una comprobación del nivel que se ha alcanzado durante el proceso de enseñanza y aprendizaje, en el cual a mi me da la pauta para poder tomar acciones consecuentes a lo cual yo estoy programado, el hecho de la comprobación me ayudan unas herramientas que yo predetermino de acuerdo a las condiciones y de acuerdo a las personas a quien yo me estoy dirigiendo.

Además, de los profesores entrevistados, uno entiende la evaluación como retroalimentación, uno como acreditación y uno como exploración. Pero, de 
todas las formas de entender la evaluación no hay alusión a la evaluación entendida como apreciación, valoración, reflexión, cualificación, emisión de juicio o como toma de decisiones.

En un segundo momento, siempre con respecto a la concepción de evaluación, pero referida a su naturaleza, algunos profesores entienden la evaluación como un proceso, otros la entienden como una herramienta o instrumento, e incluso otros la entienden como una acción. Es decir, también existe una dispersión en la forma de entender la naturaleza de la evaluación.

¿Una acción? El grupo más significativo lo constituye la noción de evaluación como una acción, como un acto, posiblemente único y aislado, más que como una actividad realizada en acciones sucesivas. Al definir la acción de evaluar, usan verbos en infinitivo, pero de diferente índole y con diferentes significados. Usan verbos como explorar, conocer, saber, verificar, cuantificar y medir (los conocimientos adquiridos por el alumno).

¿Un proceso? Ciertamente algunos la definen como un proceso, aunque no clarifican cuáles son sus fases ni su orden de sucesión. La definen como un proceso sin dar más detalles de lo que implica dicho proceso. Un profesor la entiende como un proceso dentro de un proceso más grande llamado proceso de enseñanza-aprendizaje. Un profesor ubica la evaluación como el final de un proceso.

Proceso que sirve para varias cosas, cuánto ha avanzado el alumno, hacer un diagnóstico cuáles son sus saberes, momento culmen de un proceso de E-A para verificar diferente situaciones dentro de diferentes procesos.

La evaluación para mí es un proceso.

... es como un proceso, que sirve para varias cosas, primero para saber cuánto el alumno ha avanzado, ha aprendido en un proceso de enseñanza aprendizaje, ...

¿Herramienta o instrumento? Una tercera creencia, la menos consistente, no entiende la evaluación como proceso ni como acción, sino como herramienta o instrumento. Entienden que la evaluación es un instrumento o una herramienta que usan los profesores para obtener datos y medir.

Las creencias de los profesores universitarios sobre evaluación 56. del aprendizaje
Yo la veo como una herramienta que permite medir el nivel de aprendizaje. 
... pero en el fondo también la concibo como un instrumento que permite la concretización misma de aprendizaje de todo tipo

Un caso aislado entiende la evaluación como una estrategia o una estrategia metodológica (para evaluar). Aún hay otro caso en el que se define la evaluación de forma indeterminada, no como proceso o herramienta, sino ano como una forma de (medir).

Esta forma de entender la naturaleza de la evaluación, como acción, proceso, o herramienta, o el mismo hecho que prevalezca la idea de evaluación como una acción aislada, ilustra la dispersión conceptual de la evaluación y pone de manifiesto la falta de claridad que los profesores tienen con respecto a su naturaleza procesual.

¿Evaluar qué? El objeto de la evaluación El objeto de la evaluación lo constituye aquello que se evalúa, lo que se evalúa en un proceso de enseñanzaaprendizaje. Para el caso del presente estudio, se encontró que de acuerdo a los profesores, lo que se evalúa cae en tres categorías:

El conocimiento: Un grupo muy significativo de profesores expresan que con la evaluación se busca medir, cuantificar o comprobar los conocimientos (adquiridos o asimilados por el estudiante). El conocimiento como objeto de la evaluación es una creencia muy consistente en los profesores.

Lo asimilado o aprendido / avances y logros: Otro grupo, igualmente significativo, expresa una ambigüedad o una indeterminación con respecto al objeto de la evaluación y se limitan a expresar que se mide, cuantifica o verifica lo aprendido o lo asimilado lo adquirido y lo captado (por el estudiante) y lo enseñado (por el profesor). También usan los términos avances y logros del estudiante como objeto de la evaluación; es decir, que entienden que lo que se busca medir o verificar a través de la evaluación son los logros y avances. Esta creencia también es muy consistente por parte de los profesores.

Al expresar en forma genérica e indeterminada el objeto de la evaluación, se carece de indicadores y pistas para identificar si lo aprendido, asimilado, enseñado, o los logros son contenidos u objetivos, conocimientos o destrezas; y por tanto pueden ser todos ellos, ninguno de ellos o sólo algunos de ellos.

Los objetivos de aprendizaje En una categoría muy disminuida aparecen dos profesores que afirman que lo que se mide es el alcance o el avance de los objetivos. En todo caso la referencia directa al objeto de la evaluación es el alcance y avance, y los objetivos vienen a ser una referencia indirecta 0 aclarativa que tampoco da pistas sobre el tipo de aprendizaje implicado. 
Evaluación es una forma de verificar si el conocimiento que se quiso transmitir realmente se transmitió en el alumno, verificar si realmente el alumno tiene ese conocimiento

... medir en qué grado se ha asimilado el conocimiento.

Parte de lo señalado antes, en una asignatura queremos saber qué tanto los estudiantes han avanzado

La evaluación se mide sistemáticamente de lo aprendido.

Cuantificar en alguna medida los conocimientos adquiridos por los estudiantes.

Conocer por medio de diferentes metodologías que tanto ha aprendido el estudiante en "X" materia.

No hay alusión a que el objeto de la evaluación sean aptitudes, actitudes, competencias, habilidades, destrezas, hábitos u otro tipo de conductas cognitivas, afectivas o psicomotores.

Si se reconstruye la concepción de evaluación del aprendizaje de los profesores usando sus palabras y consistencia de su uso, quedaría expresado de la siguiente manera:

Tabla 1: Conceptualización de la evaluación del aprendizaje por parte de los profesores. El tamaño de la letra representa la consistencia en el uso del término por parte del profesor.

\begin{tabular}{|c|c|c|c|c|}
\hline & Es un/una & $\begin{array}{l}\text { Con Sentido/ } \\
\text { propósito de }\end{array}$ & $\begin{array}{l}\text { Objeto de la } \\
\text { evaluación }\end{array}$ & Agente \\
\hline 1 & acción de & cuantificar & $\begin{array}{l}\text { el conocimiento } \\
\text { (asimilado, adquirido, } \\
\text { captado, aprendido) }\end{array}$ & $\begin{array}{l}\text { por /del /en el } \\
\text { estudiante }\end{array}$ \\
\hline 2 & proceso de & medir & lo aprendido & \\
\hline 3 & forma de & verificar & el aprendizaje & \\
\hline 4 & herramienta para & comprobar & los objetivos & \\
\hline 5 & instrumento para & acreditación & lo alcanzado & \\
\hline 6 & estrategia para & retroalimentación & el avance & \\
\hline 7 & comprobación de & & $\begin{array}{l}\text { lo asimilado / } \\
\text { captado / enseñado }\end{array}$ & \\
\hline
\end{tabular}




\section{El proceso de enseñanza-aprendizaje}

Vinculadas a la concepción de evaluación hay otros términos y conceptos que aparecen a lo largo de sus intervenciones y opiniones en forma consistente. De hecho son tan consistentes que develan sus significados con bastante claridad. Esos conceptos son los de enseñanza, aprendizaje y proceso enseñanzaaprendizaje.

Enseñanza $=$ Transmisión $\quad$ Los profesores entienden la enseñanza como "transmisión de conocimientos". La noción develada es la de enseñanza entendida como la actividad intencional del profesor con la cual se pretende que el estudiante adquiera, asimile o aprenda los conocimientos que éste cree que el estudiante necesita o los que están plasmados en el programa/ planificación. No hay una alusión a la enseñanza diferente de esta.

Aprendizaje $=$ Asimilación $\quad$ Por otro lado, los profesores entrevistados entienden el aprendizaje como "una asimilación de conocimientos". Noción que es reforzada por otras palabras similares como captación y adquisición de conocimientos.

PEA $=$ Modelo tradicional Dentro del proceso enseñanza-aprendizaje también se puede evidenciar que los profesores entienden el proceso enseñanza-aprendizaje como un proceso de transmisión de conocimientos por parte del profesor y un proceso de asimilación o adquisición de conocimientos por parte del alumno. Por las evidencias y indicios implícitos en las opiniones de los profesores se puede afirmar que la noción de proceso de enseñanzaaprendizaje latente en sus creencias y conocimiento profesional se identifican con características, roles, actuaciones y nociones de los agentes propios del modelo tradicional y con un modelo de educación bancaria. Se asocia al profesor con el depositario del conocimiento o la fuente del conocimiento, que luego dicho conocimiento puede ser transmitido al estudiante, y que en éste se puede almacenar. Suena a una actividad mecánica, causal e infalible donde lo que se enseña se asimila.

Estos son algunas las palabras de los profesores que explicitan las nociones enseñanza, aprendizaje y que permiten construir la concepción de modelo de enseñanza-aprendizaje que prevalece en ellos:

Evaluación es una forma de verificar si el conocimiento que se quiso transmitir realmente se transmitió en el alumno.

... vamos a trazarnos unos objetivos a esa línea nos vamos a enfocar, y a esa línea nos tenemos que guiar realmente y ahí depende del 
docente como es que va a lograr transmitir lo que quiere transmitir en este caso, un tema, un conocimiento o lo que sea, pero al final para mí la evaluación es verificar si ese proceso tuvo éxito o no en el alumno.

... tratamos no solo de medir en qué grado se ha asimilado el conocimiento, sino que también tratamos de ver, si el alumno tiene la capacidad de alcanzar esa acreditación que se ha planteado,

En un segundo momento verificar, si lo que dado ha sido asimilado.

... queremos ver que tanto el estudiante ha logrado captar sobre la parte que corresponde a esa asignatura.

Cuantificar en alguna medida los conocimientos adquiridos por los estudiantes.

... medir el conocimiento que ha adquirido un estudiante o cualquier persona en un proceso educativo, eso es.

\section{El sentido de la evaluación}

A los profesores también se les preguntó sobre el sentido que le daban a la evaluación; es decir, sí la evaluación tenía algún sentido, y si lo tenía, cuál creían que era. Las respuestas a esta interrogante resultan ser muy dispersas y no permiten estructurar categorías consistentes dado que prácticamente cada profesor le da un sentido diferente a la evaluación. Entre la variedad de creencias que despliegan los profesores, le dan a la evaluación el sentido de control, sondeo, verificación, corroboración, seguimiento, propósito, resultados, medición, orientación y garantía. Algunos expresaron lo que ellos creían era el sentido en forma ambigua o indeterminada. Otros, no expresaron el sentido que ellos le encontraban a la evaluación.

Aunque sí es necesario destacar que hay un pequeño grupo de profesores que le dan a la evaluación el sentido de verificación o corroboración. Ellos creen que la evaluación tiene sentido sí ésta sirve para verificar o corroborar los conocimientos que los alumnos han adquirido, la enseñanza del profesor o el desarrollo de la planificación. Aunque es necesario dejar claro que los profesores no tienen criterio unificado ni una tendencia única o lo suficientemente robusta con respecto al sentido que tiene la evaluación. Algunos ejemplos de de sus comentarios son: la verificación de los conocimientos de los estudiantes o lo que el 
estudiante ha alcanzado hasta cierto periodo de lo que yo he tratado de transmitirle y en otra parte también creería, que puedo utilizar la misma evaluación para verificar los procesos de enseñanza que yo estoy utilizando.

... el sentido que tiene la evaluación siempre es de corroborar lo que yo estoy impartiendo, si esta enseñanza se tiene que adaptar, se tiene que modificar o se tiene que superar,...

Para mí, el sentido de la evaluación va en la línea de la planificación que tenés de la materia o el tema que estás dando o lo que sea en ese sentido y te permite corroborar si vas bien en la línea del logro de los objetivos planeados, el sentido va íntimamente relacionado con el objetivo a mi juicio, ...

yo creo que el sentido de la evaluación es cerrar el lazo, para controlar el asunto, ... hay diferentes modos de evaluación que yo creo que el sentido de todo eso es controlar lo que estamos haciendo, digo yo.

... yo más bien veo en la línea, el sentido lo tiene en los resultados, es decir, que siempre en toda evaluación no espero solamente una nota, sino qué resultados me va a generar todo ese proceso, es decir, que es lo que yo quiero alcanzar con los muchachos, sino que yo como docente, hasta qué punto he logrado alcanzar lo que me he propuesto en el curso. Entonces yo creo que el sentido de la evaluación está más precisamente en la línea del resultado que queremos esperar tanto del alumno como parte del docente.

Pienso que son competencias, cognitivas, procedimentales y actitudinales, eso es, el sentido de la evaluación es medir las competencias.

Yo lo vería en el sentido que nos ayuda a garantizar, si tiene un fin, ahí estamos todos de acuerdo, garantizar los conocimientos que va adquiriendo el estudiante, ya que como universidad tenemos que certificar que el estudiante ha adquirido cierto nivel, ese es el fin que yo le veo.

\section{El propósito de la evaluación}

Los profesores también presentan dispersión en sus opiniones con respecto al propósito de la evaluación del aprendizaje. Los profesores creen que el propósito de la evaluación es: para asegurar el nivel de logro de aprendizaje, 
para descubrir errores y retroalimentar (2 profesores), para ver el cumplimiento de metas de la planificación, para acompañar el PEA, para calificar, para diagnosticar el nivel de aprendizaje adquirido, para poner en práctica el conocimiento adquirido (3 profesores), para reflexionar, para retroalimentar mi enseñanza y para coaccionar al estudiante.

En todo caso, la preocupación de la verificación del nivel de aprendizaje o conocimiento alcanzado está siempre presente.

Yo creo quizás que la evaluación va más enfocada a asegurarnos hasta qué nivel ha alcanzado de aprendizaje el estudiante, qué tanto pudo captar, qué tanto el pudo aplicar, cuánto conocimiento ha adquirido, y este, en un momento dado lo pueda utilizar para posteriores materias. Siento que es una forma de obtener información, el nivel de conocimiento y si aprendió realmente.

Pues en mi caso, yo lo veo como para hacer un diagnóstico de ese nivel de aprendizaje que han adquirido los estudiantes, que tanto ha funcionado mi metodología, yo como docente para que ellos adquieran conocimientos y para aplicarles una evaluación me doy cuenta de cuánto ellos han aprendido, cuanto han comprendido para aplicar también esos temas

También aparece la idea de la retroalimentación tanto para el estudiante en su aprendizaje como para el profesor en su enseñanza.

También yo creo que es para descubrir los errores que está cometiendo y luego nosotros le indicamos mira en esto es lo que estas fallando.

Mi pensamiento, para mí, la evaluación sería como categorizar dentro del grupo de alumnos a los mismos alumnos, a verificar los errores que han cometido, entonces a ese alumno, si yo quiero un estándar, quiero tener como una línea, cualquiera que sea el alumno el que después de la evaluación alcanzó ese nivel, es satisfactorio, pero aquellos en los cuales que no han logrado llegar, para mí la evaluación me sirve para poner atención a ellos y seguir trabajándolos hasta llegar ahí.

Pues en mi caso, yo lo veo como para hacer un diagnóstico de ese nivel de aprendizaje que han adquirido los estudiantes, que tanto ha funcionado mi metodología, yo como docente para que ellos adquieran conocimientos y para aplicarles una evaluación me doy cuenta de cuánto ellos han aprendido, cuanto han comprendido 
para aplicar también esos temas. Esto me da como referencia a mí también de poder cambiar mi metodología. Porque esto puede ser que también eso influya en el aprendizaje de ellos, por eso lo decía desde un principio, la evaluación yo la veo desde los dos aspectos.

Y también entienden que uno de los propósitos de la evaluación es ver o asegurarse que los conocimientos aprendidos son puestos en práctica.

Pues en mi caso, yo lo veo como para hacer un diagnóstico de ese nivel de aprendizaje que han adquirido los estudiantes, que tanto ha funcionado mi metodología, yo como docente para que ellos adquieran conocimientos y para aplicarles una evaluación me doy cuenta de cuánto ellos han aprendido, cuanto han comprendido para aplicar también esos temas.

Para mí la evaluación debería de servir como un proceso de reflexión que el alumno al final sepa aplicar lo que ha aprendido y esto obviamente me va a servir a mí para poder determinar si la metodología que yo he utilizado ha sido la correcta.

\section{Conclusión}

Poniendo en perspectiva los hallazgos de la presente investigación, se pueden establecer varios elementos inferenciales sobre las creencias que los profesores tienen sobre evaluación.

El primero es que hay suficiente evidencia para afirmar que prevalece un sistema de creencias, -de naturaleza afectiva, adquiridas en la vida cotidiana y en la experiencia-, sobre la evaluación de los aprendizajes; y que por tanto, el conocimiento profesional o base teórica sobre la evaluación, de naturaleza eminentemente cognitiva, producto de una formación pedagógica-docente, está poco presente en sus opiniones y valoraciones profesionales.

También, según la evidencia, en los profesores prevalece una concepción de evaluación del aprendizaje como una acción de cuantificación del conocimiento adquirido por el estudiante. La cuantificación hace referencia directa a una medición o cálculo matemático que proporciona un promedio y se expresa en un número o cantidad al cual se le denomina nota o calificación. Entienden los profesores que el objeto de la evaluación es el conocimiento.

Por otro lado, los profesores no tienen claridad sobre el sentido de la evaluación. Les resulta difícil identificar cual es el sentido que tiene la evaluación del aprendizaje dentro del proceso educativo. Esto queda demostrado en la 
dispersión y fragmentación de sus opiniones con respecto al sentido, ya que prácticamente cada profesor le da a la evaluación su propio sentido.

Algo muy parecido sucede con el propósito de la evaluación. Hay una gran dispersión de opinión sobre el propósito que le asignan a la evaluación que es prácticamente sacar una tendencia. Además, los argumentos que dan para explicar el sentido de la evaluación son los mismos -o muy parecidos- para identificar el propósito de la evaluación.

Se evidencia un reduccionismo en la comprensión de la evaluación del aprendizaje por parte de los profesores. Ese reduccionismo se manifiesta cuando los profesores usan las mismas palabras e ideas para conceptualizar la evaluación, para explicar su sentido y para asignarle el propósito. Las nociones de medir, cuantificar, verificar, conocimiento, están presentes en las tres dimensiones de la evaluación aquí investigadas (concepción, sentido y propósito).

Se evidencian algunas contradicciones en el pensamiento del docente. Por un lado, en el grupo focal donde se plantean preguntas abiertas y donde el profesor tiene la oportunidad de expresarse espontanea y libremente con sus verdaderos pensamientos y creencia, ellos expresan opiniones más propias. Pero en el instrumento con preguntas con alternativas, donde ellos tienen la oportunidad de hacer valoraciones por contrastación (con respecto a las alternativas propuestas), los profesores recurren al deber ser de la evaluación y de su práctica y así consignan su respuesta. De aquí que, la concepción de evaluación, por ejemplo, en el grupo focal las respuestas son espontaneas y muy suyas y proporcionan una información que no se corrobora con la respuesta a la misma pregunta hecha en el cuestionario. Para el caso, en el cuestionario, el $70 \%$ de los profesores dicen que el aprendizaje es una construcción del conocimiento que es tanto subjetivo como social, cuando en el grupo focal entendían el aprendizaje como una asimilación. O la concepción de enseñanza donde en el cuestionario la identifican como un proceso de facilitación por parte del profesor para que el estudiante aprenda por sí mismo, y ya en el grupo focal lo habían definido como transmisión. Todo esto evidencia que los profesores no tienen conocimientos sólidos y consistentes sobre evaluación y que en contextos más controlados -con opciones especificas- su pensamiento se deja guiar por el deber ser.

\section{Referencias}

Alonso, M. y otros. (1995). Concepciones docentes sobre la evaluación en la enseñanza de las ciencias. Monografía de Enseñanza de las Ciencias. Alambique, 5. 
Bouvet, J. F. (1999). Hierro en las espinacas... y otras creencias. Madrid: Grupo Santillana de Ediciones.

Carr, W. y Kemmis, S. (1988). Teoría Crítica de la Enseñanza, Barcelona: Editorial Martínez de Roca.

Contreras, S. (2009). Creencias curriculares y creencias de actuación curricular de los profesores de ciencias chilenos. Revista Electrónica de la Enseñanza de las Ciencias, 8(2), 505-526.

Dewey, J. (1933). Cómo pensamos. Cognición y desarrollo humano. Barcelona: Paidós.

Dilts, R. (1996). Las creencias. Barcelona: Ediciones Urano

Dilts, R. (1997). Cómo cambiar creencias. Barcelona: Sirio.

Faria, E. (2008). Creencias y matemáticas. Cuadernos de Investigación y Formación en Educación Matemática, 3(4), 9-27.

Durán, E. (2001). Las creencias de los profesores: un campo para deliberar en los procesos de formación. Acción Educativa. Revista Electrónica. http:// uas.uasnet.mx/cise/rev/Num1.

Flores, P. (1998). Concepciones y creencias de los futuros profesores sobre las matemáticas, su enseñanza y aprendizaje. Investigación durante las prácticas de enseñanza. Granada: Mathema.

Gallego, Ma J. (1991). Investigación sobre pensamientos del profesor: aproximaciones al estudio de las teorías y creencias de los profesores. Revista Española de Pedagogía, 189, 287-325.

García, L; Azcárate, C. y Moreno, M. (2006). Creencias, concepciones y conocimiento profesional de profesores que enseñan cálculo diferencial a estudiantes de ciencias económicas. Relime, 9(1), 85-116.

Gómez-Chacón, I. (2003). La tarea intelectual de las matemáticas Afecto, metaafecto y los sistemas de creencias. Boletín de la Asociación Matemática Venezolana, 10(2), 225-247.

Gómez, L. (2008). Los determinantes de la práctica educativa. Universidades, 58(38), 29-39.

González, M. (2000). I Concepciones sobre la evaluación del aprendizaje. Pedagogía Universitaria, 5(2), 1-32.

Jackson, P. (2002). Práctica de la enseñanza. Buenos Aires: Editorial Amorrortu.

López, R. (2001). Creencias del profesorado universitario sobre evaluación. Tesis doctoral Universidad de Granada Facultad de Ciencias de la Educación

Martínez, M.; Del Pozo, M.; Vega, R.; Varela, M.; Fernández, M. y Guerrero, A. (2001). ¿Qué pensamiento profesional y curricular tienen los futuros profesores de ciencias de secundaria? Enseñanza de las Ciencias, 19(1), 67-87.

McKay, M. y Fanning, P. (1993). Prisioneros de la creencia. Madrid: Los libros del comienzo.

Mendoza, A. (1998). Conceptos y creencias de la evaluación docente. Revista Interuniversitaria de Formación del Profesorado, 33, 107-120. 
Mora, F. y Barrantes, H. (2008) ¿Qué es matemática? Creencias y concepciones en la enseñanza media costarricense. Cuadernos de investigación y formación en educación matemática 3(4), 71-81

Moreno, M., Azcárate, G. (2003). Concepciones y creencias de los profesores universitarios de matemáticas acerca de la enseñanza de las ecuaciones diferenciales. Enseñanza de las Ciencias, 21(2), pp. 265-280.

Pajares, F. (1993). Preservice teachers' beliefs: A focus for teacher education. Action in Teacher Education, 15(2), 45-54.

Pajares, F. (1992). Teacher's beliefs and education research: cleaning up a messy construct. Review of Educational Research 62(3), 307-332.

Ponte, J. (1999). Las creencias y concepciones de maestros como un tema fundamental en formación de maestros. En Krainer, K. \& Goffree, F. (Eds.) On research in teacher education: From a study of teaching practices to issues in teacher education (pp. 43-50). Traducción (resumida) de Casimira López.

Prieto, M. (2008). Creencias de los profesores sobre Evaluación y Efectos Incidentales. Revista de Pedagogía, 29(84), 123-144.

Prieto. M. y Contreras, G. (2008). Las concepciones que orientan las prácticas evaluativas de los profesores: un problema a develar. Estudios pedagógicos, 34(2), 245-262.

Richardson, V. (1996). The role of attitudes and beliefs in learning to teach. In J. Sikula (ed.): Handbook o research on teacher education (102-119). Nueva York: MacMillan.

Rico, L. (1995). Conocimientos y creencias de los profesores de matemáticas sobre evaluación. Granada: Servicio de Reprografía de la Facultad de Ciencias. Universidad de Granada.

Serrano, R. (2010). Pensamiento del profesor: un acercamiento a las creencias y concepciones sobre el proceso de enseñanza-aprendizaje en la educación superior. Revista de Educación, 352, 267-287.

Shulman, L. (1996). Capítulo I Paradigmas y programas de investigación en el estudio de la enseñanza: una perspectiva contemporánea. En Wittrock, M. La investigación de la enseñanza, I Enfoques, teorías y métodos (pp. 9-68). Barcelona: Paidós Educador.

Thompson, A. (1992). Teachers' beliefs and conceptions: a synthesis of the research, en Grouws, D. (ed.), Handbook of research on mathematics teaching and learning, pp. 127-146. Nueva York: Macmillan.

Vergara, C. (2011). Concepciones de evaluación del aprendizaje de docentes destacados de educación básica. Revista Actualidades Investigativas en Educación, 11(1), 1-30. 